\title{
Perioperative Hyperchloremia Is Associated With Postoperative Acute Kidney Injury in Patients With off-Pump Coronary Artery Bypass Grafting: A Retrospective Study
}

\author{
Yang Wang, MD*, Ruhua Shen, MD*, Xinya Li, MD*, Haimiao Jiao, MD, Zhi Li, MD, Jianjun Ge, MD \\ Department of Cardiovascular Surgery, The Southern District of the First Affiliated Hospital of University of Science and \\ Technology of China
}

\section{ABSTRACT}

Background: The relationship between perioperative hyperchloremia and postoperative acute kidney injury (AKI) is not well established. Our study aimed to evaluate the association between perioperative hyperchloremia and acute kidney injury in patients undergoing off-pump coronary artery bypass grafting (CABG).

Methods: Patients with coronary disease who underwent off-pump CABG between April 2017 and December 2019 were enrolled in this retrospective study. The patients with perioperative hyperchloremia were matched 1:1 to patients without perioperative hyperchloremia. The primary outcome was the postoperative acute kidney injury rate. The secondary outcomes included intensive care unit (ICU)-free days, postoperative hospitalization days, wound infection rate, and in-hospital mortality. Propensity score matching and univariate and multivariate logistic regression analyses were used in this study.

Results: A total of 321 patients who underwent offpump CABG were included in the analysis. Propensity score matching selected 83 pairs for the final comparison. The results showed that the postoperative AKI rate was significantly different between the hyperchloremia and no hyperchloremia groups $(56.6 \%$ versus $15.7 \% ; P<.001)$. The number of ICU-free days, postoperative hospitalization days, wound infection rate, and in-hospital mortality were similar between the two groups. In the multivariable, logistic regression analysis, hyperchloremia was independently associated with the development of postoperative AKI (odds ratio [OR] $=1.814,95 \%$ confidence interval $[\mathrm{CI}]:$ 1.072-3.070, $P=.026)$.

Received November 1, 2020; accepted November 13, 2020.

* These authors contributed equally to this work.

Correspondence: Fianjun Ge, MD, Department of Cardiovascular Surgery, The Southern District of the First Affiliated Hospital of University of Science and Technology of China, Zhengwu District, Hefei 230036, China (e-mail: abslyyxzdxgwk@163.com).
Conclusions: Perioperative hyperchloremia is associated with an increase in the postoperative AKI rate among patients undergoing off-pump CABG.

\section{INTRODUCTION}

Acute kidney injury (AKI) in patients who undergo cardiothoracic, brain or abdominal surgery is associated with a higher mortality rate, longer hospital stay, increased medical expenses, and lower rates of long-term survival [Dasta 2008; Hobson 2015; Hobson 2009; Korenkevych 2016]. In previously published studies, the incidence of AKI, which is independently associated with worse midterm survival during cardiac surgery, is as high as $84.2 \%$ [Chou 2019]. Determining the risk factors for the development of postoperative AKI in cardiac surgical patients may help avoid this profound complication. Many studies have examined the perioperative risk factors associated with postoperative AKI [Kumar 2011; Vives 2014]. There are some well-known independent risk factors for AKI following cardiac surgery, including old age, body mass index (BMI), the duration of cardiopulmonary

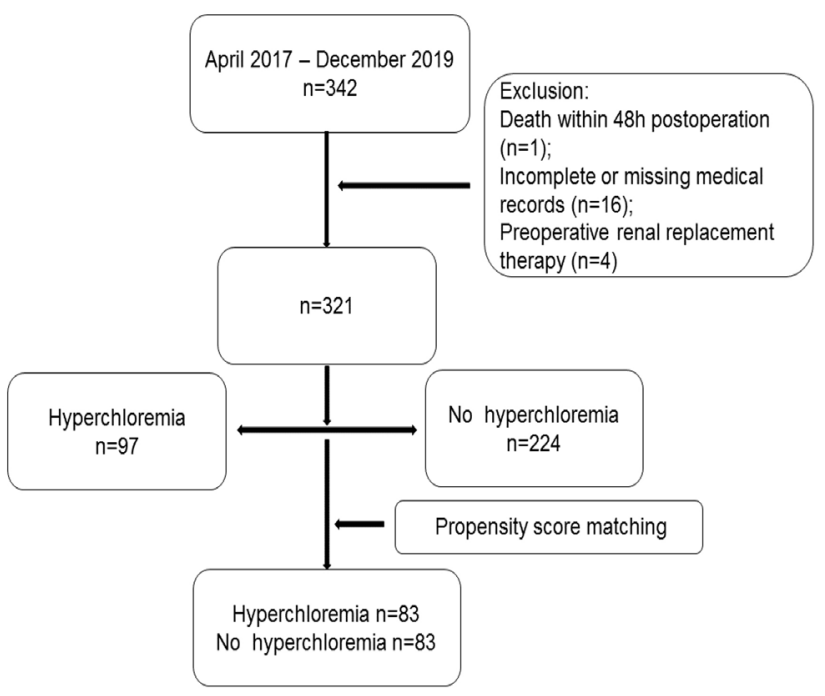

Flow chart for patient selection. 
Table 1. Patients' perioperative characteristics in the unmatched (a) and matched groups (b)

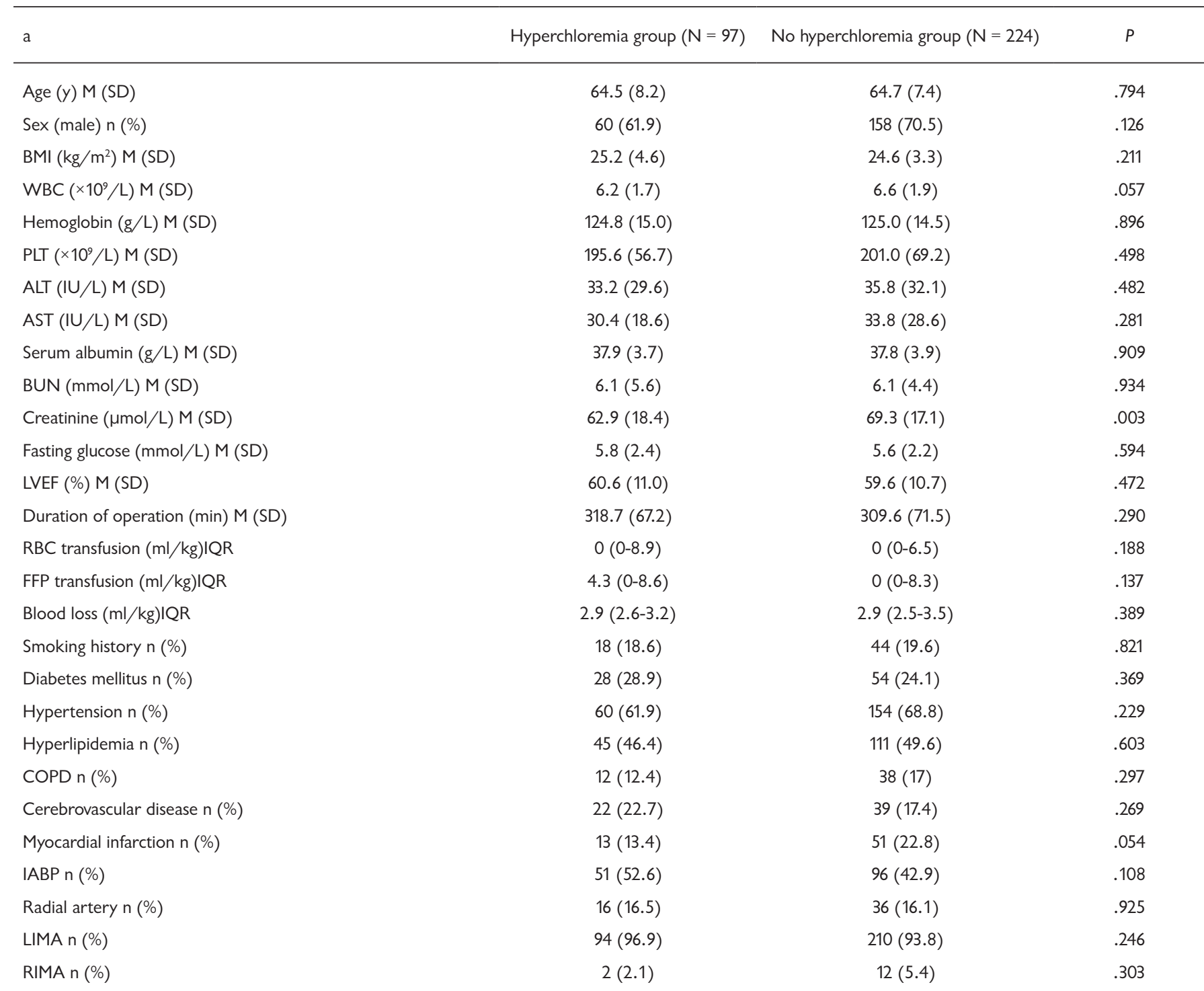

bypass (CPB), hypertension, reduced left ventricular ejection fraction, and impaired preoperative renal function [Chou 2019; Kumar 2011]. However, the exact mechanism of AKI after cardiac surgery is not completely understood.

Studies have shown that hyperchloremia or chlorideliberal fluids are a potential risk factor for AKI after liver surgery, septic shock, and brain surgery [Nadeem 2014; Oh 2019; Suetrong 2016]. Serum chloride plays an important role in determining the tonicity of plasma and influences the acid-base balance. Balanced crystalloids, $0.9 \% \mathrm{NaCl}$, or blood products often are used during the perioperative period in cardiac surgery, disrupting the balance of the serum chloride concentration. Studies have suggested that hyperchloremia may impair renal function by decreasing the renal blood flow velocity and renal cortical tissue perfusion [Chowdhury 2012; Yunos 2010]. Nevertheless, there is limited available information regarding the role of the perioperative chloride concentration in patients undergoing coronary artery bypass grafting (CABG) and whether hyperchloremia is associated with the development of AKI in this population.

In this study, we investigated the relationship between perioperative hyperchloremia and postoperative AKI in patients undergoing off-pump CABG. The primary outcome was the development of perioperative AKI. The number of ICU-free days, postoperative hospitalization days, wound infection rate, and in-hospital mortality were the secondary outcomes.

\section{METHODS}

Patients and study design: This was a single-center, retrospective, propensity matched cohort study. Patients who underwent elective off-pump CABG surgery in the southern district of the First Affiliated Hospital of USTC between April 2017 
Table 1. Patients' perioperative characteristics in the unmatched (a) and matched groups (b)

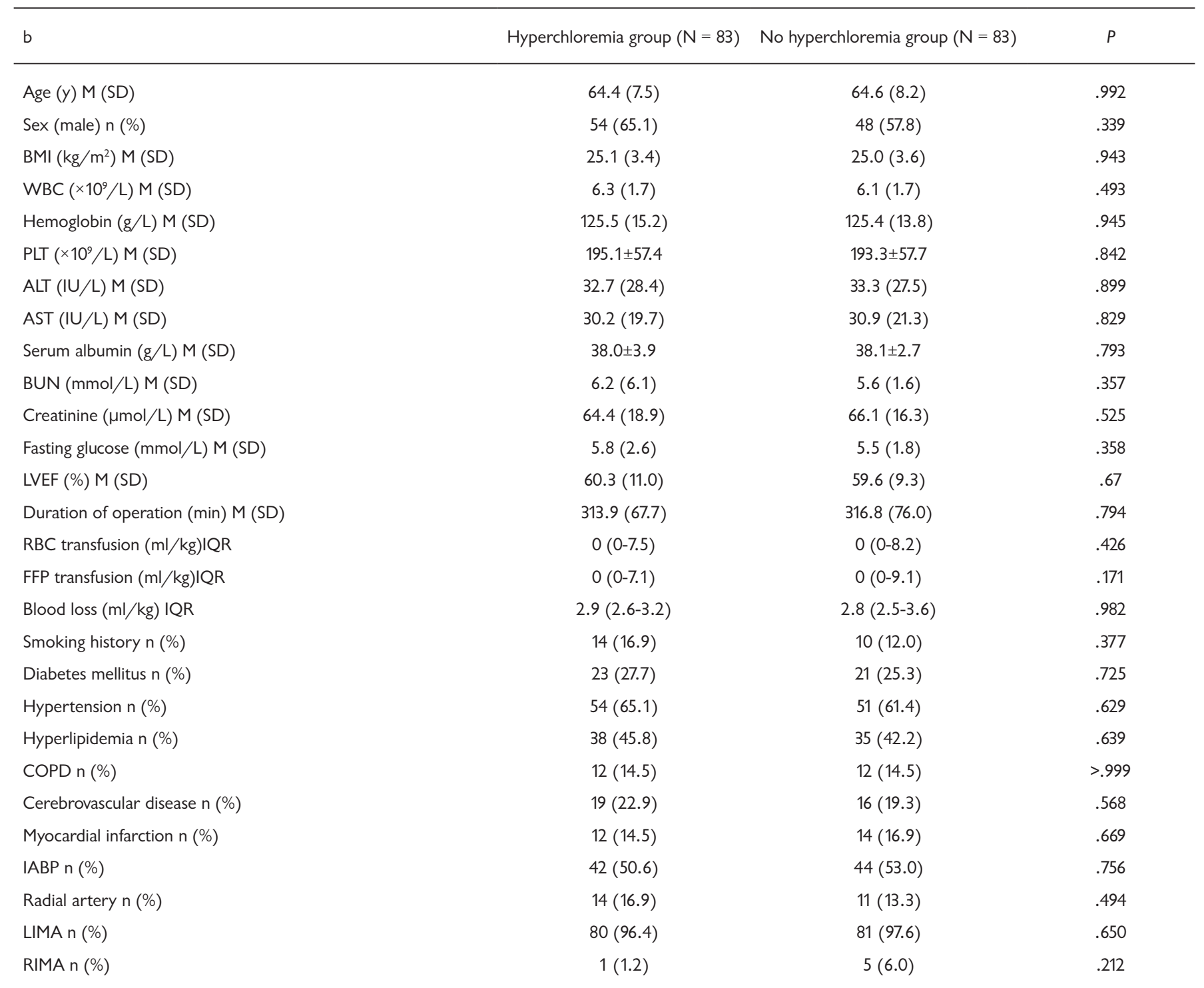

and December 2019 retrospectively were evaluated. The exclusion criteria were as follows: (1) other concomitant surgical procedures, (2) lack of accurate records of the main demographic and clinical characteristics, surgical details, and postoperative outcomes, (3) death within 48 hours postoperation, (4) emergency surgery, and (5) preoperative renal replacement therapy. Prior to analysis, the patient's information was anonymized and deidentified. The risks and benefits of the surgery were explained to all patients, who signed informed consent forms before surgery.

Surgical treatment: All off-pump CABG surgeries were performed by an experienced cardiovascular surgery team under general anesthesia with standard median sternotomy. All patients achieved complete revascularization. After surgery, the patients were transferred to the cardiovascular intensive care unit and underwent daily routine laboratory tests during postoperative days (PODs) 0 to 2 . The patients were extubated when they could breathe spontaneously, achieved adequate blood gases, and had stable hemodynamics.

Study variables: The demographic and clinical characteristics, surgical details, and postoperative outcomes were obtained by retrospectively reviewing the medical charts. Perioperative serum creatinine and electrolyte levels were collected by routine perioperative laboratory testing. The baseline creatinine and chloride levels were defined as the levels measured most recently in blood sampled from a vein within 1 month before surgery. The postoperative creatinine levels $(\mu \mathrm{mol} / \mathrm{L})$ and chloride levels $(\mathrm{mmol} / \mathrm{L})$ were measured at 24 hours and 48 hours after surgery. Urine output may be affected by diuretic or vasopressor was not used.

Postoperative AKI was defined as a categorical variable, according to the AKIN criteria as any increase in postoperative serum creatinine of $0.3 \mathrm{mg} / \mathrm{dL}(26.5 \mathrm{~mol} / \mathrm{L}) 48 \mathrm{~h}$ of postoperation or a more than $50 \%$ increase from the preoperative baseline 
Table 2. Outcomes of the unmatched (a) and matched (b) groups

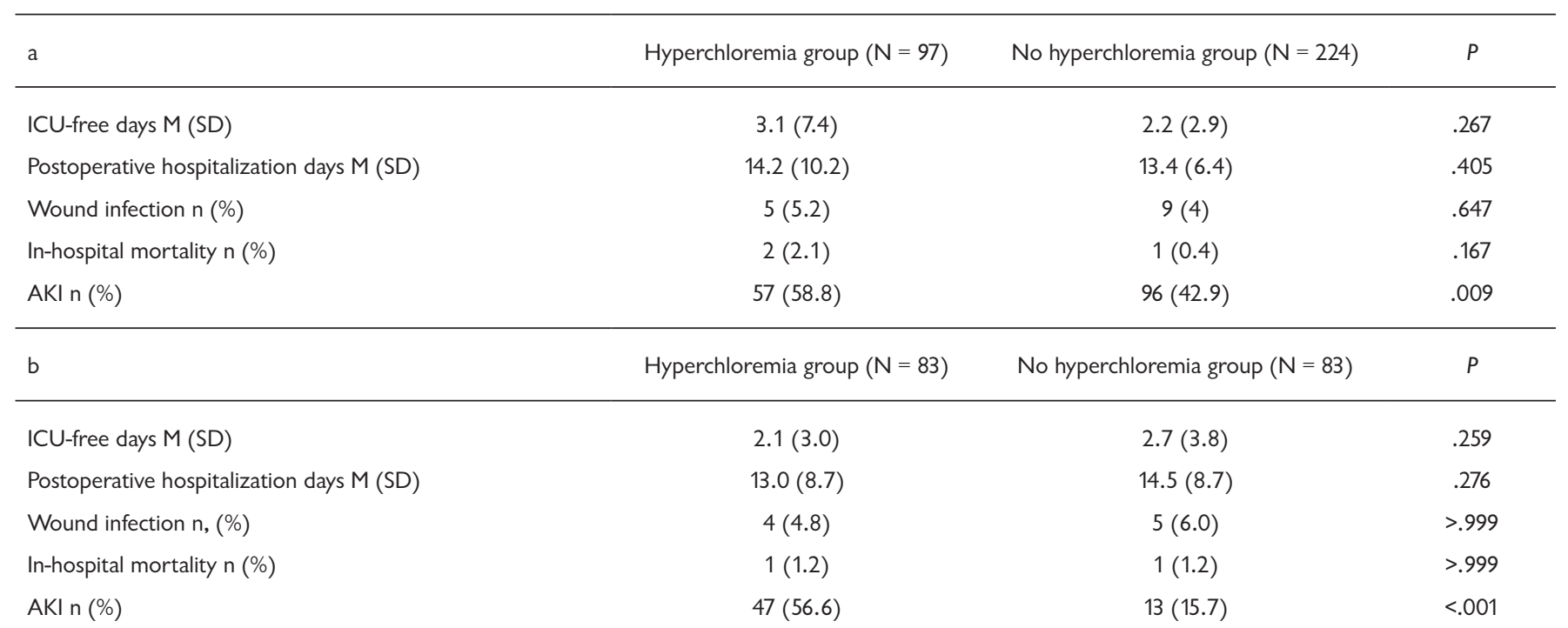

serum creatinine level [Mehta 2007]. Perioperative hyperchloremia was defined as a perioperative $\mathrm{Cl}->110 \mathrm{mmol} / \mathrm{L}$.

Statistical analyses: Demographic and clinical data were compared between patients with perioperative hyperchloremia and those without perioperative hyperchloremia. Continuous data are presented as the mean \pm standard deviation (SD) or medians with ranges, and categorical data are presented as percentages. Continuous outcomes were compared by the Student's t-test or Mann-Whitney $\mathrm{U}$ test. The $\chi^{2}$ test and Fisher's exact test were performed to compare categorical variables. Because the two groups appeared different at baseline, we used propensity scores based on logistic regression to match patients who had perioperative hyperchloremia and those who did not. The variables of age, sex, BMI, LVEF, laboratory data (white blood cell (WBC) count, hemoglobin, platelet (PLT), serum albumin, creatinine), surgical details (duration of operation, red blood cell (RBC) transfusion, fresh frozen plasma (FFP) transfusion, blood loss, intra-aortic blood pump (IABP) use), smoking history, and comorbidities (diabetes mellitus, hypertension, hyperlipidemia, chronic obstructive pulmonary disease (COPD), cerebrovascular disease, history of myocardial infarction) were used to create the propensity score. Univariate and multivariate logistic regression analyses were performed to evaluate independent predictors for postoperative AKI. Independent variables with $P<.2$ in the univariate models were incorporated as covariates in the multivariate regression model. All statistical analyses were carried out using SPSS version 33 (SPSS, IBM Corporation). $P$ values $<.05$ were considered statistically significant.

\section{RESULTS}

Patient characteristics: Between April 2017 and December 2019, 342 patients underwent off-pump CABG (Figure). Sixteen patients were removed from this study because of a lack of medical records. One patient who died within 48 hours postoperation was excluded. Four patients were excluded because of preoperative renal replacement therapy. A total of 321 patients who underwent off-pump CABG were included in the analysis. As shown in Table 1, before propensity score matching, the preoperative creatinine was different between the hyperchloremia group and the no hyperchloremia group $(P<.003)$ (Table 1$)$. After propensity score matching, 83 pairs of patients were selected. These baseline characteristics and laboratory data were not significantly different.

Propensity score matching for the analysis of outcomes: Before propensity score matching, the number of ICU-free days, postoperative hospitalization days, wound infection rate and in-hospital mortality were not different between the two groups (Table 2). In the hyperchloremia group, the incidence of postoperative AKI was higher than that in the no hyperchloremia group (58.8\% versus $42.9 \%, P=.009)$. In the propensity score-matched groups, the rate of AKI in the hyperchloremia group was significantly higher than that in the no hyperchloremia group $(56.6 \%$ versus $15.7 \%, P<.001)$. The other outcomes were not significantly different between the two groups.

Risk factors for postoperative AKI development: We divided the patients with and without postoperative AKI to ascertain the risk factors for postoperative AKI development. Table 3 presents the outcomes of the univariate and multivariate logistic regression analysis for the occurrence of postoperative AKI (Table 3). The covariates chosen from the univariate analysis with a $P<.2$ additionally were analyzed in the multivariate logistic regression analysis. After univariate analysis, age (OR 1.039, $P=.008$ ), duration of operation (OR $1.004, P=.025)$, fasting glucose (OR 1.151, $P=.015)$, RBC transfusion (OR 1.062, $P=0$ ), FFP transfusion (OR 1.046, $P=.006)$, hyperchloremia (OR 1.9, $P=.009$ ), diabetes mellitus (OR 1.685, $P=.044$ ), hypertension (OR 1.404, $P=.156$ ), smoking history (OR 1.549, $P=.125$ ), and cerebrovascular disease (OR 1.492, $P=.162$ ) were subjected to multivariate 
Table 3. Univariate and multivariate analysis of the occurrence of postoperative acute kidney injury

\begin{tabular}{|c|c|c|c|c|}
\hline Sex & $1.265(0.790-2.027)$ & .328 & & \\
\hline BMI & $1.015(0.957-1.076)$ & .623 & & \\
\hline PLT & $1(0.996-1.003)$ & .891 & & \\
\hline Hemoglobin & $0.996(0.981-1.011)$ & .625 & & \\
\hline Serum albumin & $0.972(0.917-1.030)$ & .331 & & \\
\hline BUN & $1.02(0.969-1.072)$ & .452 & & \\
\hline Creatinine & $1.002(0.989-1.014)$ & .806 & & \\
\hline Diabetes mellitus & $1.685(1.015-2.796)$ & .044 & $1.225(0.663-2.262)$ & .517 \\
\hline Hypertension & $1.404(0.879-2.242)$ & .156 & $1.407(0.828-2.392)$ & .207 \\
\hline Hyperlipidemia & $0.804(0.518-1.247)$ & .330 & & \\
\hline Smoking history & $1.549(0.886-2.707)$ & .125 & $2.295(1.249-4.219)$ & .007 \\
\hline COPD & $1.351(0.738-2.474)$ & .33 & & \\
\hline Cerebrovascular disease & $1.492(0.851-2.615)$ & .162 & $1.265(0.677-2.364)$ & .461 \\
\hline Myocardial infarction & $1.039(0.601-1.798)$ & .89 & & \\
\hline IABP & $1.282(0.825-1.991)$ & .269 & & \\
\hline Duration of operation & $1.004(1.000-1.007)$ & .025 & $1.002(0.998-1.006)$ & .247 \\
\hline
\end{tabular}

logistic regression analysis. The multivariate logistic regression showed that age (OR 1.040, 95\% CI, 1.010-1.075), fasting glucose (OR 1.155, 95\%CI, 1.011-1.320), RBC transfusion (OR 1.062, 95\% CI, 1.014-1.111), hyperchloremia (OR 1.814, 95\% CI, 1.072-3.070), and smoking (OR 2.295, $95 \%$ CI, 1.249-4.219) were independent risk factors.

\section{DISCUSSION}

We conducted a retrospective study to evaluate the association between perioperative hyperchloremia and the development of AKI in patients after off-pump CABG. In a propensity score matching analysis, patients who had perioperative hyperchloremia had higher rates of postoperative AKI. The number of ICU-free days, postoperative hospitalization days, wound infection rate, and in-hospital mortality were similar in patients with or without perioperative hyperchloremia. By univariate and multivariate logistic regression analyses, perioperative hyperchloremia was shown to be an independent risk factor for postoperative AKI.

In our study, the incidence of hyperchloremia, which is one of the most common electrolyte abnormalities after offpump CABG, 48 hours after surgery was $30.2 \%$. Several previous studies reported a positive correlation between serum chloride or hyperchloremia and adverse clinical outcomes, including AKI. Marttinen et al demonstrated that elevated time-weighted chloride values were independently associated with AKI in critically ill patients [Marttinen 2016]. In a retrospective cohort study, Tak Kyu Oh et al reported that preoperative hypochloremia was related to an increased risk for postoperative acute kidney injury in patients who underwent noncardiac surgery [Oh 2019]. Kim et al reported that hyperchloremia was associated with increased incidences of both AKI and ICU mortality in ICU patients, and hyperchloremia 48 hours after ICU admission was an independent predictor for both AKI within the first 7 days of ICU admission and ICU mortality [de Vasconcellos 2018]. Bandarn et 
al reported that hyperchloremia is common in severe sepsis and septic shock and independently associated with AKI [Suetrong 2016]. Tak Kyu Oh et al also showed that perioperative hyperchloremia was associated with an increased risk of postoperative AKI after craniotomy for primary brain tumor resection [Oh 2019].

However, there are inconsistencies. A previous study by Tak Kyu Oh et al has reported that exposure to perioperative hyperchloremia is not associated with postoperative AKI in surgical ICU patients. After further analysis, they found that in patients with moderate-to-severe chronic kidney disease (stage $\geq 3$ ), a substantial perioperative increase in serum chloride levels may reflect a higher risk of AKI [Oh 2018]. Therefore, the relationship between hyperchloremia and AKI remains controversial.

Our study showed that 153 patients (47.7\%) had postoperative AKI within the first 48 hours after the operation, and 168 patients $(52.3 \%)$ did not. The incidence of postoperative $\mathrm{AKI}$ in our research remains high. Identifying the risk factors for postoperative AKI can benefit patients during cardiac surgery. There are some well-known preoperative, intraoperative, and postoperative risk factors, including diabetes, hypertension, obesity, older age, elevated preoperative creatinine level, CPB time, and the use of postoperative inotropic drugs [Chen 2016]. However, there are few studies on the relationship between serum chloride and AKI in patients undergoing cardiac surgery. Kimura et al found that there was no significant relationship between postoperative chloride concentration and AKI in pediatric patients after congenital cardiac surgery [Kimura 2019]. Considering that the variables included in the author's multivariate logistic regression may have collinearity, the strength of the conclusions may be limited. In a prospective sequential study, David McIlroy et al reported that a perioperative fluid strategy to restrict intravenous chloride administration was not associated with an altered incidence of AKI or other metrics of renal injury in adult patients undergoing cardiac surgery [McIlroy 2017]. The relevant data and subgroup analyses of off-pump CABG have not been sent to us. Lomivorotov et al indicated that the use of chlorine-containing fluids ( $7.2 \% \mathrm{NaCl} / 6 \%$ HES 200/0.5) does not increase the incidence of AKI in patients after onpump CABG [Lomivorotov 2014]. However, the relationship between chlorine load and hyperchloremia still needs further study. David McIlroy et al found that the incidence of perioperative hyperchloremia also reached $60 \%$ under the chlorine restriction strategy [McIlroy 2017]. This suggests that factors other than administered chloride load may play an important role in the development of hyperchloremia.

To the best of our knowledge, this is the first retrospective study to show the relationship between perioperative hyperchloremia and postoperative AKI in patients with off-pump CABG. As our primary outcome, the incidence of postoperative AKI in the hyperchloremia group was significantly higher than that in the no hyperchloremia group, regardless of matching. In clinical practice, we may need to be aware of the adverse effects of hyperchloremia on the renal function of perioperative patients. We also investigated the number of ICU-free days, postoperative hospitalization days, wound infection rate, and in-hospital mortality rate as secondary outcomes. Although there were no significant differences in the secondary outcomes between the two groups before and after matching, the number of ICU-free days, postoperative hospitalization days, wound infection rate, and in-hospital mortality rate were high in the hyperchloremia group compared with those in the no hyperchloremia group before matching.

Although promising, our study has several important limitations. First, this is a retrospective and single-center study, and the number of enrolled patients was small, thereby limiting the strength of the conclusions drawn by our study. Large-scale multicenter prospective studies are required. Second, propensity score adjustments can only be limited to known confounding variables. Some unknown confounding variables may still influence the results. Although the authors aimed to collect and adjust data related to the potential risk factors for AKI in coronary heart disease patients after offpump CABG, there might be other confounders, such as sodium, that may contribute to the development of AKI. Third, we did not separate the AKI stage levels in this study because of the small proportion of patients with stage 2 or 3 AKI. For this reason, we did not investigate the association between hyperchloremia and the severity of AKI. Fourth, although we included patients with preoperative chronic kidney disease in this study, because of the small sample size, we did not sub-analyze these patients who have a high risk of AKI after surgery. Fifth, we did not use urine volume as a diagnostic AKI criterion in this study, which may limit the strength of the article's conclusions, but the validity of this criterion in the context of cardiac surgery is still not very clear [McIlroy 2013].

\section{CONCLUSIONS}

Perioperative hyperchloremia was associated with the development of postoperative AKI in patients who underwent off-pump CABG. Perioperative hyperchloremia might be a potential independent risk factor for postsurgical AKI in patients who underwent off-pump CABG.

\section{REFERENCES}

Chen SW, Chang CH, Fan PC, et al., 2016. Comparison of contemporary preoperative risk models at predicting acute kidney injury after isolated coronary artery bypass grafting: a retrospective cohort study. BMJ Open 6: e010176.

Chou YH, Huang TM, Wu VC, et al. 2019. Associations between preoperative continuation of renin-angiotensin system inhibitor and cardiac surgery-associated acute kidney injury: a propensity score-matching analysis. J Nephrol 32: 957-966.

Chowdhury AH, Cox EF, Francis ST, Lobo DN. 2012. A randomized, controlled, double-blind crossover study on the effects of 2-L infusions of $0.9 \%$ saline and plasma-lyte(R) 148 on renal blood flow velocity and renal cortical tissue perfusion in healthy volunteers. Ann Surg 256: 18-24.

Dasta JF, Kane-Gill SL, Durtschi AJ, Pathak DS, Kellum JA. 2008. Costs and outcomes of acute kidney injury (AKI) following cardiac surgery. 
Nephrol Dial Transplant 23: 1970-4.

de Vasconcellos K, Skinner DL. 2018. Hyperchloraemia is associated with acute kidney injury and mortality in the critically ill: A retrospective observational study in a multidisciplinary intensive care unit. J Crit Care 45: $45-51$

Hobson C, Ozrazgat-Baslanti T, Kuxhausen A, et al. 2015. Cost and Mortality Associated With Postoperative Acute Kidney Injury. Ann Surg 261: $1207-14$

Hobson CE, Yavas S, Segal MS, et al. 2009. Acute Kidney Injury Is Associated With Increased Long-Term Mortality After Cardiothoracic Surgery. Circulation 119: 2444-2453.

Kimura S, Iwasaki T, Shimizu K, et al. 2019. Hyperchloremia Is Not an Independent Risk Factor for Postoperative Acute Kidney Injury in Pediatric Cardiac Patients. J Cardiothorac Vasc Anesth 33: 1939-1945.

Korenkevych D, Ozrazgat-Baslanti T, Thottakkara P, et al. 2016. The Pattern of Longitudinal Change in Serum Creatinine and 90-Day Mortality After Major Surgery. Ann Surg 263: 1219-1227.

Kumar AB, Suneja M. 2011. Cardiopulmonary Bypass-associated Acute Kidney Injury. Anesthesiology: The Journal of the American Society of Anesthesiologists 114: 964-970.

Lomivorotov V, Fominskiy E, Efremov S, et al. 2014. Infusion of 7.2\% $\mathrm{NaCl} / 6 \%$ Hydroxyethyl Starch 200/0.5 in On-Pump Coronary Artery Bypass Surgery Patients: A Randomized, Single-Blind Pilot Study. Shock (Augusta, Ga.) 41: 193-9.

Marttinen M, Wilkman E, Petaja L, Suojaranta-Ylinen R, Pettila V, Vaara ST. 2016. Association of plasma chloride values with acute kidney injury in the critically ill - a prospective observational study. Acta Anaesthesiol Scand 60: 790-9.

McIlroy DR, Argenziano M, Farkas D, Umann T, Sladen RN. 2013. Incorporating Oliguria Into the Diagnostic Criteria for Acute Kidney Injury After On-Pump Cardiac Surgery: Impact on Incidence and Outcomes. Journal of Cardiothoracic and Vascular Anesthesia 27: 1145-1152.
McIlroy D, Murphy D, Kasza J, Bhatia D, Wutzlhofer L, Marasco S. 2017. Effects of restricting perioperative use of intravenous chloride on kidney injury in patients undergoing cardiac surgery: the LICRA pragmatic controlled clinical trial. Intensive Care Med 43: 795-806.

Mehta RL, Kellum JA, Shah SV, et al. 2007. Acute Kidney Injury Network: report of an initiative to improve outcomes in acute kidney injury. Crit Care 11: R31.

Nadeem A, Salahuddin N, El Hazmi A, et al. 2014. Chloride-liberal fluids are associated with acute kidney injury after liver transplantation. Crit Care 18: 625.

Oh TK, Do SH, Jeon YT, Kim J, Na HS, Hwang JW. 2019. Association of Preoperative Serum Chloride Levels With Mortality and Morbidity After Noncardiac Surgery. Anesthesia \& Analgesia 129: 1494-1501.

Oh TK, Kim CY, Jeon YT, Hwang JW, Do SH. 2019. Perioperative Hyperchloremia and its Association With Postoperative Acute Kidney Injury After Craniotomy for Primary Brain Tumor Resection: A Retrospective, Observational Study. J Neurosurg Anesthesiol 31: 311-317.

Oh TK, Song IA, Kim SJ, et al. 2018. Hyperchloremia and postoperative acute kidney injury: a retrospective analysis of data from the surgical intensive care unit. Crit Care 22: 277.

Suetrong B, Pisitsak C, Boyd JH, Russell JA, Walley KR. 2016. Hyperchloremia and moderate increase in serum chloride are associated with acute kidney injury in severe sepsis and septic shock patients. Crit Care 20: 315 .

Vives M, Wijeysundera D, Marczin N, Monedero P, Rao V. 2014. Cardiac surgery-associated acute kidney injury. Interact Cardiovasc Thorac Surg 18: 637-45.

Yunos NM, Bellomo R, Story D, Kellum J. 2010. Bench-to-bedside review: Chloride in critical illness. Crit Care 14: 226. 